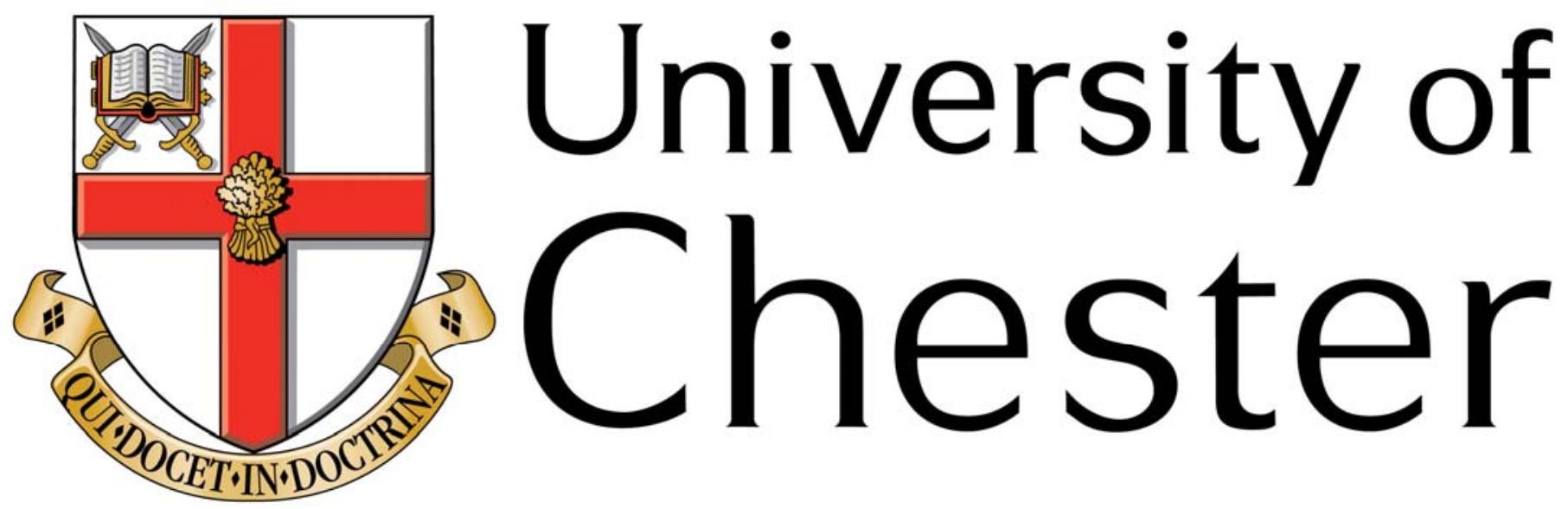

This work has been submitted to ChesterRep - the University of Chester's online research repository

\title{
http://chesterrep.openrepository.com
}

Author(s): Stephen J Lewis

Title: Morphological aspects of male and female hands

Date: 1996

Originally published in: Annals of Human Biology

Example citation: Lewis, S. J. (1996). Morphological aspects of male and female hands. Annals of Human Biology, 23(6), 491-494

Version of item: Author's post-print

Available at: http://hdl.handle.net/10034/42054 


\title{
SHORT REPORT
}

\section{Morphological aspects of male and female hands}

\author{
Stephen Lewis \\ University College Chester, Chester, UK
}

Address for correspondence: Stephen Lewis PhD, MA, BSc, University College Chester, Cheyney Road, Chester CH1 4BJ, UK.

Summary: A series of hand radiographs from Gwynedd, North Wales, was assessed for frequencies in digital and metacarpal formulae - the relative projection of the fingers and knuckles respectively. While the digital formula showed a sexual dimorphism, the metacarpal formula did not. Males showed a single most frequent digital formula ( $3>4>2>5>1$ : $69.6 \%$ ) while females showed two almost equally frequent forms $(3>2>4>5>1: 44.1 \%, 3>4>2$ $>5>1: 42.3 \%$ ). For the metacarpal formula, however, females showed a single most frequent form $(2>3>4>5>1$ : $53.1 \%)$ while males showed two almost equally frequent forms $(2>3>4>5>1$ : $40.6 \%, 3>2>4>5>1$ : $39.2 \%$ ). Digital and metacarpal formulae are morphological variables which may also have functional significance in the understanding of how certain hand forms may be ill-fitted for certain tasks.

\section{Introduction}

Classification of the human hand into different types, based upon the relative projection of the fingers, was first noted by Ecker (1875) although Wood Jones (1920) is credited with the neologism 'digital formula' (Napier 1993). Despite the role which hand form may have as a marker for other physical conditions (Garn, Hertzog, Poznanski and Nagy 1972, Poznanski, Garn, Nagy and Gall 1972, Schaumann and Kimura 1991), the digital formula has received only sporadic attention (George 1930, Phelps 1952, Blincoe 1959, 1962, Kimura 1965). Numbering the digits from thumb (1) to little finger (5), three digital formulae are discernible, depending upon the relative projections of each finger: $3>2>4>5>1,2>3>4>5>1$ and $3 \approx 2>4>5>1$. Wood Jones (1920) also applied the use of relative projection to the metacarpals, describing three metacarpal formulae $3>2>4>5>1,3>4>2>5>1$ and $3>4 \approx 2>5>1$. The metacarpal formula appears to have received no subsequent attention.

\section{Materials and methods}

Dorsi-palmar projection, hand radiographs of mature male and female patients were obtained from Ysbyty Gwynedd, Bangor, Wales. Using patient surname (Ashley and Davies 1966) and domicile, those uncharacteristic of or non-resident in Gwynedd were excluded from this study. The sample 
comprised a total of 217 males and 113 females.

Using the third digit as the central axis of the hand, and with the aid of a rolling ruler, each radiograph was assessed for the relative projection of the tip of the distal phalanx (for the digital formula) and the head of the metacarpal (for the metacarpal formula) in each ray. As proposed by Phelps (1952), where a difference in the projection of two digits or metacarpals was less than $1 \mathrm{~mm}$, they were deemed to be of approximately equal projection.

The frequencies of each of the digital and metacarpal formulae were tabulated (table 1) and the differences between the sexes in the relative frequencies of each pair of digital and metacarpal formulae were investigated using a $2 \times 3$ $\chi^{2}$ test.

\section{Results}

A statistically significant difference between the sexes was found in the frequencies of the three digital formulae $(p \ll 0.0001)$. A majority of males $(69.59 \%)$ showed the $3>4>2>5>1$ formula while the most frequent female type was the $3>2>4>5>1$ formula (44.14\%), although followed closely by the $3>4>2>5>1$ formula (42.34\%). In both sexes the $3>4 \approx 2>5>1$ was the least frequent (males: $9.68 \%$, females: $13.51 \%$ ).

No sex difference was evident for the metacarpal formula. Both sexes showed the $2>3>4>5>1$ metacarpal formula most frequently (males: $40.55 \%$, females: $53.15 \%$ ), followed by the $3>2>4>5>1$ formula (males: $39.17 \%$, females: $30.63 \%$ ) with the $3>4 \approx 2>5>1$ formula least frequent (males: $20.28 \%$, females: $16.22 \%)$.

\section{Conclusion}

Reviewing previous studies, George (1930) suggested that the most frequent digital formulae were $3>4>2>5>1$ for males and $3>2>4>5>1$ for females. More recently, Kimura (1965) also found that the $3>4>2>5>1$ digital formula was most frequent in Japanese and Taiwanese males and that both sexes showed this formula more frequently than in comparable studies among Americans, Canadians and the Dutch. However, unlike these latter groups, he found no differences between the sexes in the frequency of digital formulae. In the present study $3>4>2>5>1$ was also found to be the most prevalent in males (69.6\%), but while $3>2>4>5>1$ was most frequent in females (44.1\%), the $3>4>2>5>1$ form was almost equally frequent (42.3\%). This difference between the sexes was statistically significant. A significant sex difference was not evident for the metacarpal formula, although statistical significance was approached when the $3 \approx 2>4>5>1$ form was partitioned out of the $\chi^{2}$ test. It was noticeable, however, that while the $2>3>$ $4>5>1$ form was the single most frequent form among females (53.2\%), this time males showed two forms with almost equal frequency $(2>3>4>5>1$ : $40.6 \%, 3>2>4>5>1: 39.2 \%)$.

These findings accord with those of Takai (1978), who suggested that each row of bones in the hand was under separate developmental control. Although digital formulae are evident as early as the seventh fetal week (Phelps 1952), 
the relative frequencies are different from those of adults (Kimura 1965), suggesting that post-natal factors may also be involved.

Given the hand's adaptability, the functional significance of digital and metacarpal formulae is not to seek the best hand type for certain tasks, but rather to determine which hands are relatively disadvantaged in performing those tasks. Furthermore, when not ideally matched, what task-specific compensating mechanisms are invoked in the hand and arm, and what is the likely concomitant pathology. Taken together, nine different combinations of digital and metacarpal formulae are possible, allowing a greater degree of discernment between hand forms.

\section{Acknowledgements}

I thank Mrs K. D. Eaton, Superintendent Radiographer, and Dr J. G. Williams, Consultant Radiologist at Ysbyty Gwynedd for allowing me access to radiographs, and I thank Mrs A. Lewis for her help with the preparation of the manuscript. This work was supported by a University of Liverpool research development grant.

\section{References}

Ashley, D. J. B., and Davies, H. D., 1966, The use of the surname as a genetic marker in Wales. Journal of Medical Genetics, 3, 203-211.

Blincoe, H., 1959, Significant types of hands related to distal extent of digits. Anatomical Record, 133, 527-535.

Blincoe, H., 1962, Significant hand types in women according to relative lengths of fingers. American Journal of Physical Anthropology, 20, 45-48. Ecker, A., 1875, Einige Bemerkungen über einen Schwankenden Charakter in den Hand des Menschen. Archiv für Anthropologie, 8, 67-74.

Garn, S. M., Hertzog, K. P., Poznanski, A. K., and Nagy, J. M., 1972, Metacarpophalangeal length in the evaluation of skeletal malformation Radiology, 105, 375-381.

George, R., 1930, Human finger types. Anatomical Record, 46, 199-204. Kimura, K., 1965, Radiological studies on three fingers formulae. Journal of the Anthropological Society, Nippon, 72, 146-162.

Napier, J. R., 1993, Hands (Princeton, NJ: Princeton University Press), revised edition, p. 18.

Phelps, V. R., 1952, Relative index finger length as a sex-influenced trait in Man. American Journal of Human Genetics, 4, 72-89.

Poznanski, A. K., Garn, S. M., Nagy, J. M., and Gall, J. C., 1972, Metacarpophalangeal pattern profiles in the evaluation of skeletal malformations. Radiology, 104, 1-11.

Schaumann, B. A., and Kimura, S., 1991, Palmar, plantar and digital flexion creases: morphological and clinical considerations. Birth Defects: Original Articles Series, 27, 229-252.

Takai, S., 1978, Metacarpal and phalangeal lengths are influenced by row related factor. Human Biology, 50, 51-56.

Wood Jones, F., 1920, The Principles of Anatomy - as seen in the hand (London: J. \& A. Churchill). 
Table 1. Digital and metacarpal formulae: counts and frequencies.

\section{Metacarpal formulae}

\begin{tabular}{lllll} 
Digital formulae & $3>2>4>5>1$ & $2>3>4>5>1$ & $3 \approx 2>4>5>1$ & Totals \\
\hline Males & & & & \\
$3>2>4>5>1$ & $1(3.2 \%)$ & $29(13.4 \%)$ & $9(4.1 \%)$ & $45(20.7 \%)$ \\
$3>4>2>5>1$ & $32(33.2 \%)$ & $49(22.6 \%)$ & $30(13.8 \%)$ & $151(69.6 \%)$ \\
$>4 \approx 2>5>1$ & $6(2.8 \%)$ & $10(4.6 \%)$ & $5(2.3 \%)$ & $21(9.7 \%)$ \\
Totals & $85(39.2 \%)$ & $88(40.6 \%)$ & $44(20.3 \%)$ & 217 \\
Females & & & & \\
$3>2>4>5>1$ & $12(10.8 \%)$ & $30(27.0 \%)$ & $7(6.3 \%)$ & $49(44.1 \%)$ \\
$3>4>2>5>1$ & $18(16.2 \%)$ & $20(18.0 \%)$ & $9(8.1 \%)$ & $47(42.3 \%)$ \\
$3>4 \approx 2>5>1$ & $4(3.6 \%)$ & $9(8.1 \%)$ & $2(1.8 \%)$ & $15(13.5 \%)$ \\
Totals & $34(30.6 \%)$ & $59(53.2 \%)$ & $18(16.2 \%)$ & 111
\end{tabular}

